\title{
Design and Fabrication of Miniaturized Optical Chopper Operated by Electromagnetic Actuation
}

\author{
Ho Won Kim', Seong Ki Min², Young Chan Choi ${ }^{1}$, and Seong Ho Kong ${ }^{1,+}$
}

\begin{abstract}
An existing infrared (IR) analysis system is generally composed of infrared source, IR focusing lenses, IR detector, and optical chopper. An optical chopper is widely used in combination with lock-in amplifier to improve the signal-to-noise ratio by periodically interrupting incident light beam. During recent years, a few researches on miniaturized optical chopper have been reported to apply to microscaled optical systems. In this paper, a micro optical chopper operated by electromagnetic actuation is proposed and applied to a miniaturized micro-scaled optical system operating in IR spectral range. Additionally, the fabrication method of the proposed micro chopper is demonstrated. The proposed micro optical chopper is composed of the polydimethylsiloxane (PDMS) membrane, solenoid, and permanent magnet. The permanent magnet is bonded on the PDMS membrane using an ultraviolet-activated adhesive. The operation of the chopper is based on the attractive and repulsive forces between permanent magnet and solenoid induced by an electrical current flowing through the solenoid. The fabricated micro optical chopper could operate up to $200 \mathrm{~Hz}$ of frequency. The maximum operating distance of the chopper with $7 \mathrm{~mm}$ diameter membrane was $750 \mu \mathrm{m}$ at $100 \mathrm{~Hz}$ of frequency.
\end{abstract}

Keywords: Optical chopper, Infrared analysis system, PDMS

\section{INTRODUCTION}

Recently, researches on infrared (IR)-ray have been actively conducted due to a number of interesting characteristics of IR-ray [1-5]. Researches on material analysis using IR analysis system are one of the most actively studied fields because they are very exact and simple method to identify material ingredients [6-10]. However, the applications of those IR analysis system to portable equipment is limited because of its large size and complex structure. Therefore, many researches are focused on minimizing the size of the IR analysis system to broaden their applications [11].

The IR analysis system is basically composed of IR source, IR focusing lenses, IR detector, and optical chopper, as shown in Fig. 1. Many studies on partly miniaturized IR analysis system component have been reported to achieve minimizing IR analysis

\footnotetext{
${ }^{1}$ School of Electronics Engineering, Kyungpook National University, 80 Daehak-ro, Buk-gu, Daegu 702-701, Korea

${ }^{2}$ Advanced propulsion Technology Center, ADD, Yuseong P.O.Box, Daejeon, 305-600, Korea

${ }^{+}$Corresponding author: shkong@knu.ac.kr

(Received: May. 14, 2014, Revised: May. 21, 2014, Accepted: May. 22, 2014)
}

This is an Open Access article distributed under the terms of the Creative Commons Attribution Non-Commercial License (http://creativecommons.org/ licenses/bync/3.0) which permits unrestricted non-commercial use, distribution, and reproduction in any medium, provided the original work is properly cited. system $[12,13]$. Especially, researches focused on miniaturization of IR source, IR lenses and IR detector are widely reported $[14,15]$. However, despite miniaturized optical chopper is the essential part of an IR analysis system, only few researches on miniaturized optical chopper have been reported.

The optical chopper is periodically interrupting the incident IRray to prevent the temperature saturation of the IR detector or to improve the signal-to-noise ratio. The optical chopper is essential in the field using thermal characteristics of IR-ray. To miniaturize the complete IR analysis system, development of micro optical chopper is essential.

In this paper, a simple miniaturized optical chopper realized using micro machining technique is proposed. The micro optical chopper is composed of micro solenoid, $\mathrm{NdFeB}$ permanent magnet and membrane. The micro optical chopper is simply fabricated and easily integrated with a micro IR analysis system because of its

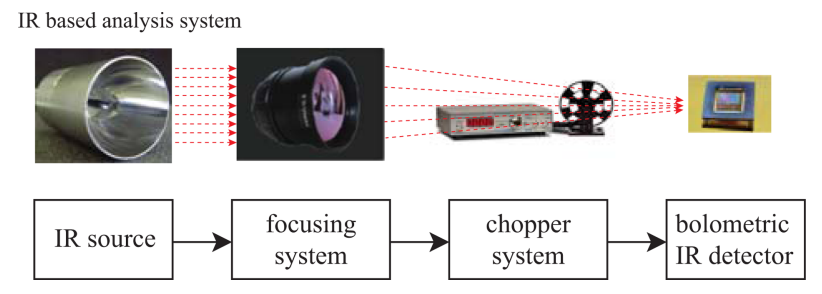

Fig. 1. Schematic diagram of an IR analysis system. 
simple structure. The proposed micro optical chopper shows a sufficient performance up to $200 \mathrm{~Hz}$ of frequency.

\section{EXPERIMENTAL}

\subsection{Design and Operation Principle}

Fig. 2 shows schematic illustration of the micro optical chopper, which is divided into membrane part and solenoid part. Solenoid part is cylindrically fabricated in order to minimize the size of the chopper. The solenoid part is composed of computer numerical control (CNC)-machined polycarbonate substrate, solenoid, and copper alloy core. The dimensions (diameter $\times$ height) of the polycarbonate substrate, solenoid, and copper alloy core are 10 $\mathrm{mm} \times 8 \mathrm{~mm}, 4 \mathrm{~mm} \times 2 \mathrm{~mm}$, and $2 \mathrm{~mm} \times 2 \mathrm{~mm}$, respectively. The solenoid is placed in the CNC-machined hole on the solenoid substrate. The membrane part is composed of permanent magnet, polydimethylsiloxane (PDMS) membrane, and polycarbonate structure. Cylindrical permanent magnet is placed and bonded on the PDMS membrane and used as actuator.

Fig. 3 shows the operating principle of the actuator as a micro optical chopper. The micro optical chopper is activated by repulsive and attractive forces induced between permanent magnet and solenoid depending on applied bias current. Fig. 4 shows an example of operation of the micro optical chopper with $7 \mathrm{~mm}$ diameter and $20 \mu \mathrm{m}$-thick membrane. Vertical actuation of permanent magnet by attractive and repulsive forces between magnet and solenoid is related with magnetic flux density around the solenoid loop. The magnetic flux density by Ampere's law is expressed in the following eq.

$$
B=\mu \times\left(\frac{n}{l}\right) \times I
$$

where $\mu$ denotes magnetic permeability of the solenoid core, $N$ is number of solenoid winding turns, $l$ is height of the solenoid, and $I$ is the bias current. Light blocking operation by raising permanent magnet attached on PDMS membrane is shown in Fig. 4 (a). The distance between permanent magnet and solenoid is increased by a repulsive force induced by an electrical positive current. Fig. 4 (b) shows light passing operation by attracting the permanent magnet to downward direction. The direction of magnetic flux density is reversed when the negative bias current is applied to the solenoid. Fig. 4 (c) shows the actuation of permanent magnet by AC current. By continuously changing the direction of the magnetic flux density around the solenoid loop by AC current, the attractive and repulsive

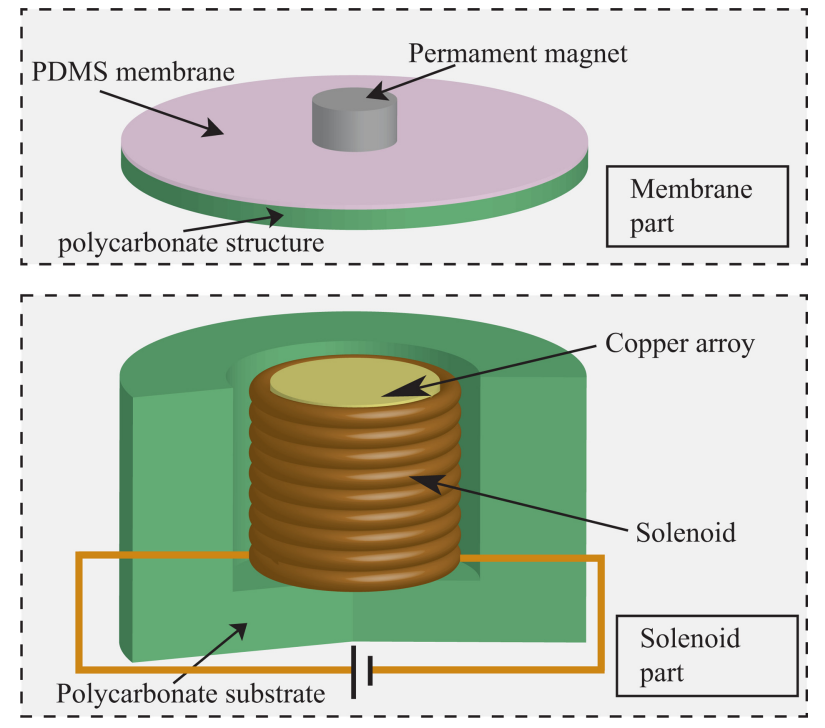

Fig. 2. Schematic illustration of the micro optical chopper.

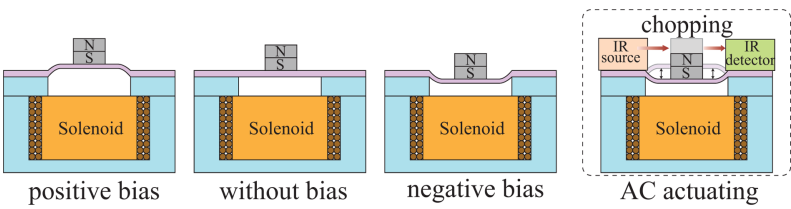

Fig. 3. Operating principle of the micro optical chopper.
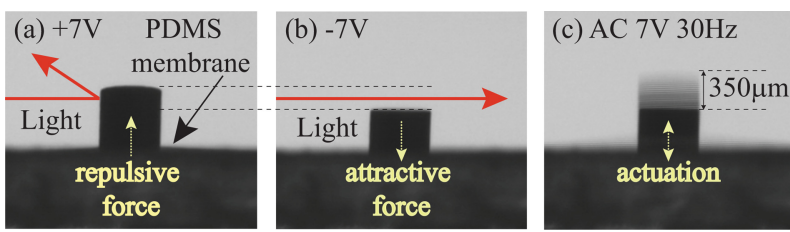

Fig. 4. Operation of the micro optical chopper with $7 \mathrm{~mm}$ diameter membrane.

forces are alternately applied to the permanent magnet. Therefore, the permanent magnet can be vibrated vertically.

\subsection{Fabrication}

Fig. 5 shows the fabrication process of the proposed micro optical chopper. The photo resist (AZ 5214) is spin-coated on a cleaned slide glass with 2000 RPM and baked for 1 minute at $110^{\circ} \mathrm{C}$. The PDMS membrane is fabricated to be $20 \mu \mathrm{m}$-thick on the slide glass by spin-coating. Photo resist is then removed by immersing the slide glass in the acetone to split the PDMS membrane. The PDMS membrane is floated in acetone when PR is removed. The floating membrane is bonded on a CNCmachined polycarbonate structure. No extra bonding process 
1. Cleaning glass using acetone

Slide glass

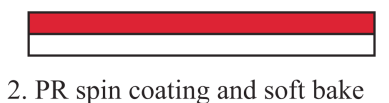

Photo resist ( 2000 RPM )

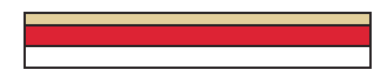

PDMS membrane

3. PDMS spin coating and bake 10 minite

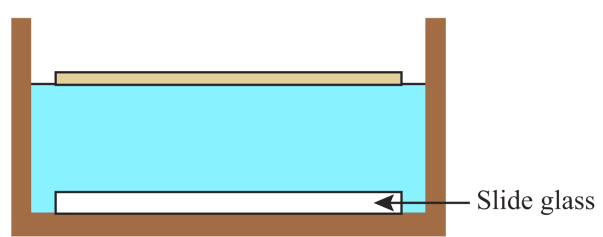

4. Remove photo resist using acetone

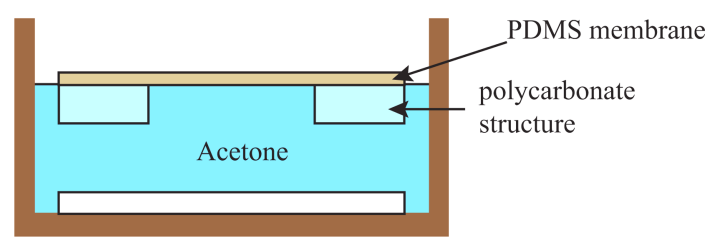

5. Bonding the floating PDMS membrane

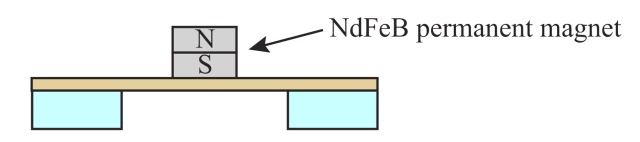

6. Dry membrane structure

and bonding with permanent magnet

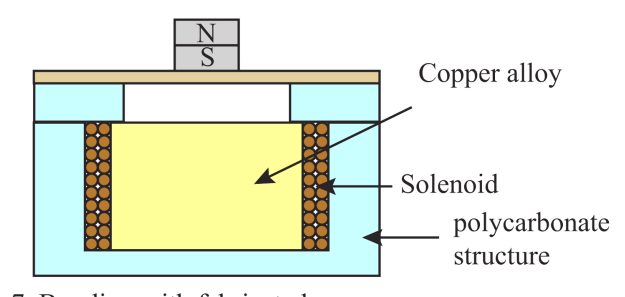

7. Bonding with fabricated poly carbonate structure

Fig. 5. Fabrication process of the micro optical chopper.

between the PDMS membrane and polycarbonate structure is necessary. They are stuck tightly to each other as acetone is naturally evaporated. The permanent magnet is bonded on the PDMS membrane using ultraviolet-activated adhesive (NOA 65, Norland, USA). By bonding the magnet on the membrane, the fabrication of the membrane part is done and ready for the following coupling process with the solenoid part.

In order to fabricate the solenoid part, a cylindrical polycarbonate substrate is $\mathrm{CNC}$ machined to have a hole at the

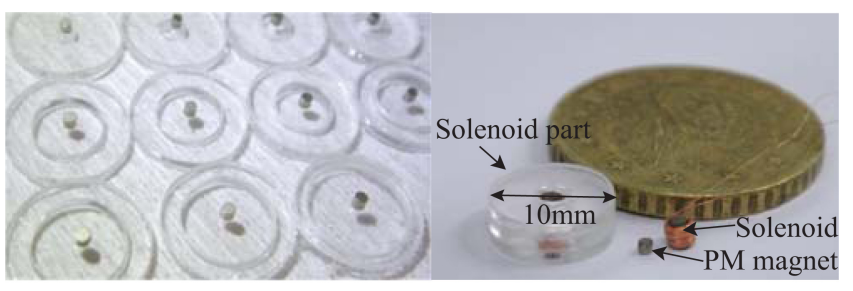

(a)

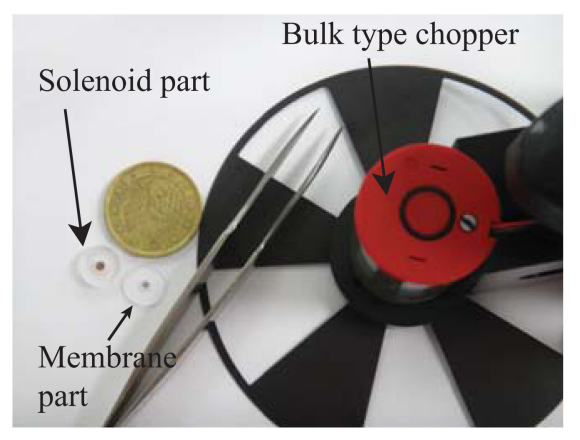

(b)

Fig. 6. (a) Fabricated membrane part and solenoid part, (b) comparison of the fabricated micro chopper to the conventional bulk-type chopper.

center to adopt the solenoid and core. The solenoid with core is then placed into the ready-made polycarbonate hole. Due to its low relative permeability $\left(1.256 \times 10^{-6} \mathrm{Hm}^{-1}\right)$ and good machinability, a copper alloy ( $90 \mathrm{wt.} \% \mathrm{Cu}, 7 \mathrm{wt} . \% \mathrm{Ag}$, and $3 \mathrm{wt} . \% \mathrm{Sn}$ ) was used as the core material for the solenoid (winding turns $=200$ ). Finally, the separately fabricated membrane part and the solenoid part are bonded with each other using a cyanoacrylate adhesive. The completed membrane and solenoid parts are shown in Fig. 6 (a). The size comparison of fabricated micro optical chopper with conventional bulk type chopper is shown in Fig. 6 (b).

\section{RESULTS AND DISCUSSIONS}

Fig. 7 shows the micro IR analysis system containing fabricated micro optical chopper. The optical fiber is used to make optical path between IR source and IR detector. By placing the chopper in optical path, the IR-ray is interrupted and it is possible to adjust the amount of incident IR-ray exposed to IR detector.

Fig. 8 shows the amount of IR-ray exposed to the IR detector depending on the on/off state of micro optical chopper. The amount of exposure is expressed as the output of the IR detector. The wavelength of used IR source is $940 \mathrm{~nm}$. At on state, the height of permanent magnet is increased by applying a repulsive force between the permanent magnet and solenoid. As a result, the 


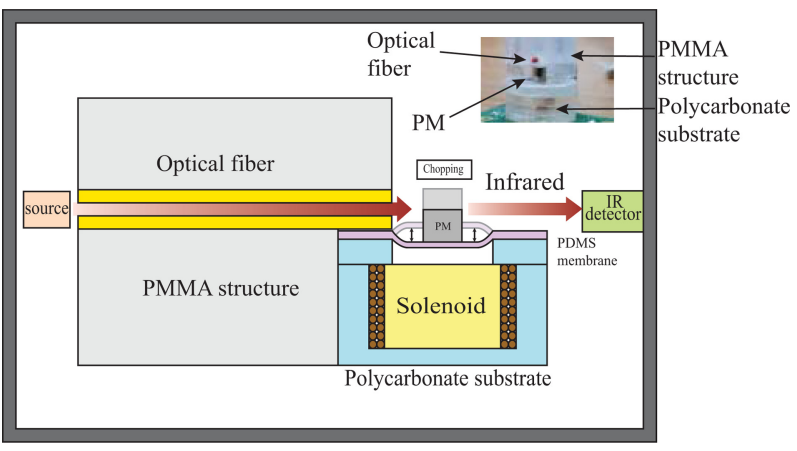

Fig. 7. Micro optical chopper system.

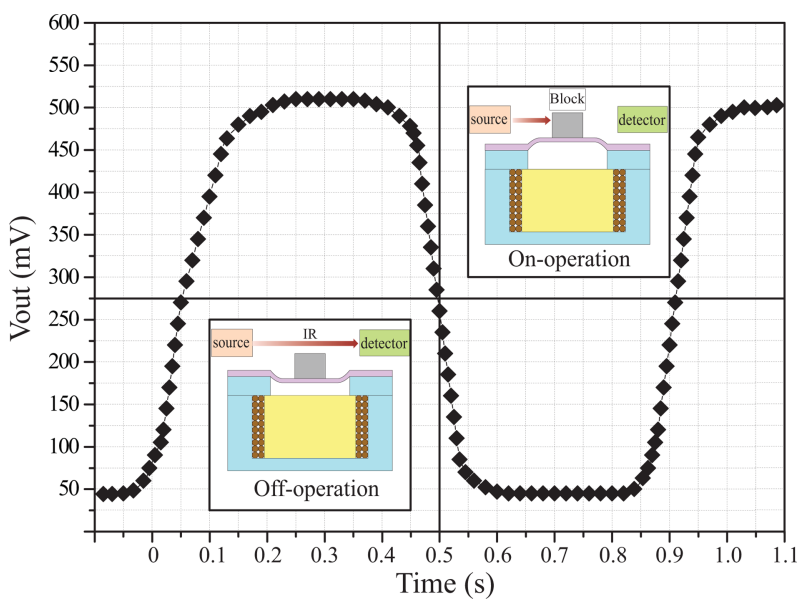

Fig. 8. The measured operational performance of the micro optical chopper.

permanent magnet placed in the IR path interrupting the IR-ray. At off state, the height of the permanent magnet is decreased by applying an attractive force between the permanent magnet and solenoid, and the entire IR-ray exposes IR detector.

Fig. 9 shows the operation distance of the micro optical chopper as a function of membrane diameter at $1 \mathrm{~Hz}$ frequency. In this measurement, the chopper shows steady operating distance at 1 $\mathrm{Hz}$ frequency. Flexible area of PDMS membrane is broadened according to the diameter of the machined hole in the polycarbonate structure. As the flexible area of PDMS membrane is increased, the operation distance of the permanent magnet can be increased at the same repulsive or attractive force.

Operating distance and number of vibration of micro optical chopper as a function of frequency are shown in Fig. 10. The micro optical chopper has almost constant operation distance at the frequency less than $50 \mathrm{~Hz}$. The operating distance rapidly increases from 50 to $100 \mathrm{~Hz}$ frequency and vibration cannot follow above $200 \mathrm{~Hz}$ frequency. This means that the micro optical chopper can operate up to $200 \mathrm{~Hz}$ frequency. The maximum

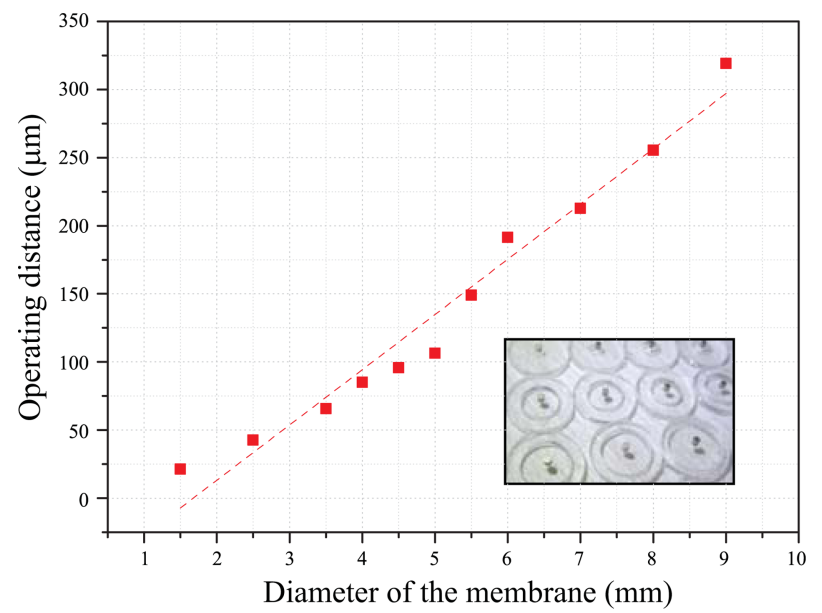

Fig. 9. Operating distance of the micro optical chopper as a function of membrane diameter at $1 \mathrm{~Hz}$ frequency.

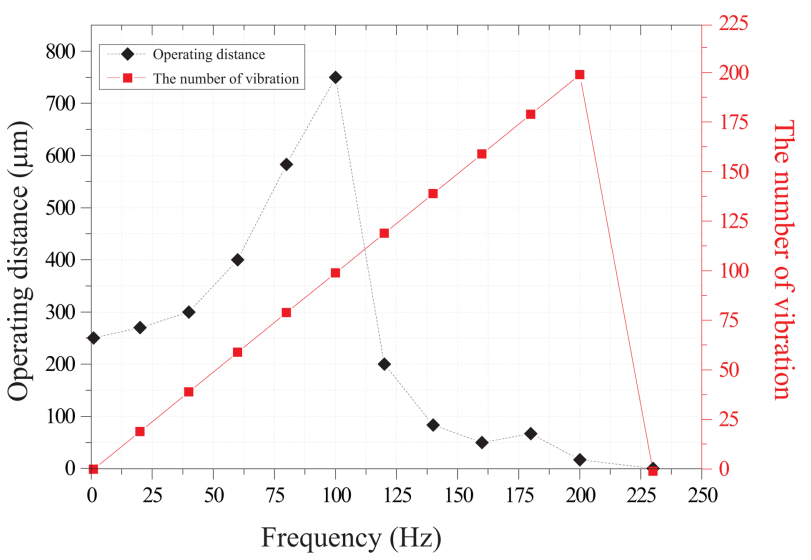

Fig. 10. Operating characteristics as a function of frequency with $7 \mathrm{~mm}$ diameter membrane.

operation range was observed at $100 \mathrm{~Hz}$ of frequency.

The bulk type chopper needs an electrical motor to operate so it suffers from expensive cost and large size. Also, it is difficult to be miniaturized and integrated in an IR analysis system. Miniaturized micro optical chopper has advantages such as a wide range of operation frequency, small size, and simple structure. The performance of micro optical chopper can be easily optimized by adjusting options such as membrane diameter and frequency. As a result of these advantages, the proposed micro optical chopper has sufficient operating performance as a chopper.

\section{CONCLUSIONS}

The conventional bulky chopper is not suitable to apply to the IR analysis system due to its large size and bulky structure. To 
resolve this issue, a miniaturized micro chopper based on an elastomeric membrane operated by electromagnetic actuation is designed and fabricated in this study. The chopper is composed of PDMS membrane, permanent magnet, solenoid, and polycarbonate structure. The polycarbonate structure is simply fabricated by CNC milling technique. The fabricated $20 \mu \mathrm{m}$-thick PDMS membrane and solenoid are combined with polycarbonate structure. Maximum operating distance is $750 \mu \mathrm{m}$ and it can be variable by adjusting diameter of PDMS membrane. The micro optical chopper can operate up to $200 \mathrm{~Hz}$ frequency. This means the minimum switching time of chopper is 0.005 second. The main advantages of miniaturized micro optical chopper are the small size, simple structure, less cost, and wide operation range of frequency compared to conventional bulk type chopper.

\section{ACKNOWLEDGMENT}

This research was financially supported by a grant to MEMS Research Center for National Defense funded by DAPA/ADD.

\section{REFERENCES}

[1] R. Jha and A. K. Sharma, "Design considerations for plasmonic-excitation based optical detection of liquid and gas media in infrared", Sens. Actuator B-Chem., Vol. 165, No. 2, pp. 271-275. 2011.

[2] C. N. Chen, "Fully quantitative characterization of CMOSMEMS polysilicon/titanium thermopile infrared sensors", Sens. Actuator B-Chem., Vol. 161, No. 1, pp. 892-900, 2012.

[3] J. C. Yang, H. Jung, G. J. Lee, J. Y. Kang, J. G. Koo, J. M. Park, K. S. Park, and S. H. Kong, "Micro-electro-mechanical-systems-based infrared spectrometer composed of multi-slit grating and bolometer array", Jpn. J. Appl. Phys., Vol. 47, No. 8, pp. 6943-6948, 2008.

[4] S. Eminoglu, M. Y. Tanrikulu, and T. Akin, "A low-cost 128 $\times 128$ uncooled infrared detector array in CMOS process", Journal of Micro ElectroMmechanical System, Vol. 17, No. 1, pp. 20-30, 2008.
[5] L. Han, X. Ming, and L. T. Liu, "A high performance uncooled a-Si TFT infrared sensor", Nano/Micro Engineered and Molecular Systems. NEMS '06. 1st IEEE International Conference on. pp. 631-634, Zhuhai, china, 2006.

[6] D. S. Tezcan, S. Eminoglu, and Tayfun Akin, "A low-cost uncooled infrared microbolometer detector in standard CMOS technology", Electron Devices, IEEE Transactions on, Vol. 50, No. 2, pp. 494-502, 2003.

[7] W. G. Baer, T. Hull, K. D. Wise, K. Najafi, and K. D. Wise, "A multiplexed silicon infrared thermal imager", Solid-State Sensors and Actuators, pp. 631-634, san Francisco, CA, USA, 1991.

[8] Y. Zhang, M. Wang, and S. Nium, "A novel small dim infrared target detecting method based on grayscale merging and connected components analysis", Modelling, Identification \& Control (ICMIC), 2012 Proceedings of International Conference on, pp. 454-459, Wuhan, Hubei, China, 2012.

[9] M. Almasri, B. Xu, and J. Castracane, "Amorphous silicon two-color microbolometer for uncooled IR detection", IEEE Sens. J., Vol. 6, No. 2, pp. 293-300, 2006.

[10] D. Takamuro, H. Takao, K. Sawada, and M. Ishida, "Highly sensitive electron-emission-type infrared sensor using a single crystalline $\mathrm{LiNb}_{3}$ ", Solid-State Sensors, Actuators and Microsystems, 2005. Digest of Technical Papers. Transducers '05. The 13th International Conference on, Vol. 1, pp. 581-584, 2005.

[11] J. Spannhake, O. Schulz, A. Helwig, and G. Muller, "Design, development and operational concept of an advanced MEMS IR source for miniaturized gas sensor systems", Sensors, 2005 IEEE, Irvine, CA, 2005.

[12] E. G. Camargo, K. Ueno, T. Morishita, M. Sato, H. Endo, M. Kurihara, K. Ishibashi, and N. Kuze, "High-sensitivity temperature measurement with miniaturized InSb mid-IR sensor", IEEE Sens. J., Vol. 7, No. 9, pp. 1335-1339, 2007.

[13] D. H. B. Wicaksono, G. Pandraud, and P. J. French, "Performance of miniaturized LPCVD-SiN membranebased 7$14 \mu \mathrm{m}$ infrared thermal detector: Analytical, modeling, and experimental study", Sensors, 2008 IEEE, pp. 498-501, lecce, Italy, 2008.

[14] J. C. Choi, J. K. Lee, Y. C. Choi, D. G. Jung, and S. H. Kong, "Infrared detector array with an incident-ray concentrator", Jpn. J. Appl. Phys., Vol. 52, 06GL12, 2013.

[15] J. T. Song, J. H. Park, J. K. Lee, J. C. Choi, and S. H. Kong, "Square-patterned narrow-band infrared emitter for filter less infrared gas sensor", Jpn. J. Appl. Phys., Vol. 51, 06GL12, 2012. 